\title{
The Dangerous Liaisons between Chronic Obstructive Pulmonary Disease and Venous Thromboembolism
}

\author{
Job Harenberg ${ }^{1,2}$ Peter Verhamme ${ }^{3}$ \\ ${ }^{1}$ Ruprecht Karls University, Heidelberg, Germany \\ 2 DOASENSE GmbH, Heidelberg, Germany \\ ${ }^{3}$ Department of Cardiovascular Sciences, Vascular Medicine and \\ Haemostasis, University of Leuven, Leuven, Belgium
}

Thromb Haemost 2020;120:363-365.

\section{Venous Thromboembolism in the Era of Comorbidities}

Acute intercurrent or chronic coexisting diseases are increasingly common in patients who are diagnosed with acute venous thromboembolism (VTE), especially in the aging population. ${ }^{1}$ These coexisting morbidities are important because they (1) increase the risk for VTE; (2) interfere with the diagnostic strategies; (3) drive the efficacy and safety of anticoagulant therapy; and (4) impact importantly on the prognosis of these patients suffering from several diseases. While this has probably been best studied for cancer-associated thrombosis, ${ }^{2}$ also chronic obstructive pulmonary disease (COPD) and VTE have a complicated relationship.

\section{COPD and the Risk of Mortality in Patients with VTE-The Tromsø Study}

In this issue, Børvik et al report on the impact of coexisting COPD on the risk of death in patients with VTE. ${ }^{3}$ The cohort consists of approximately 10,000 Tromsø inhabitants who underwent extensive phenotyping, including spirometry, and who were prospectively followed; 256 participants suffered acute VTE. The main finding of the study was that patients with preexisting COPD who were diagnosed with VTE were twice more likely to die during follow-up. The risk of death increased with the severity of COPD; crude mortality rates were $9.4 \%$ per 100 person-years in patients with normal respiratory function and skyrocketed to $50.7 \%$ per 100 person-years in patients with stage III/IV COPD. Or, the risk of death was approximately fivefold higher in patients with VTE and stage III/IV COPD compared with patients without COPD. The increased mortality was observed in both patients with pulmonary embolism (PE) and deep vein thrombosis with 7.5- and 3.8-fold higher mortality rates in patients with
Address for correspondence Job Harenberg, MD, DOASENSE GmbH, Waldhofer Strasse 102, D-69123 Heidelberg, Germany (e-mail: j.harenberg@doasense.de).

COPD stage III/IV compared with VTE patients who had a normal spirometry.

The second important finding of the study was that inactivity could not explain the increased mortality in COPD patients. As expected, the proportion of physically inactive subjects increased with the severity of COPD but inactivity was not related to the increased risk of mortality. Other risk factors for progression of COPD or VTE (such as body mass index, smoking, cancer, history of cardiovascular disease, and acute medical conditions) did also not influence mortality.

This study reminds us of the impact of coexisting COPD when patients are diagnosed with acute VTE. Half of the observed mortality was early, that is, in the first months after the diagnosis of VTE. Especially for patients diagnosed with $\mathrm{PE}$, mortality is likely to be triggered or accelerated by a compromised pulmonary circulation in patients with a poor respiratory function. There is little doubt that the casefatality of (recurrent) PE in these compromised patients is high.

In other patients, acute VTE may merely serve as a marker for the severity of the coexisting disease. Disease progression, chronic inflammation, intercurrent infections, progressive immobility, and inactivity all increase the risk of VTE but are on itself also risk factors for poor prognosis.

The poor outcome of COPD patients who suffer VTE in the Tromsø study, but also in previous studies, ${ }^{4}$ is also an important impulse to reflect on the appropriate prophylactic strategies, diagnostic algorithms that prompt timely diagnosis, and the optimal treatment on VTE in this important patient group.

\section{COPD and VTE: A Bad Marriage}

Also, the diagnosis of VTE, particularly PE, can be notoriously challenging in patients with COPD, which further underpins the importance of the prevention. Indeed, most signs and received

December 13, 2019

accepted

December 13, 2019 (c) 2020 Georg Thieme Verlag KG Stuttgart · New York
DOI https://doi.org/ $10.1055 / \mathrm{s}-0039-1701012$. ISSN 0340-6245. 
symptoms are not specific and symptoms of COPD exacerbation may mimic PE; hence, an established or presumed diagnosis of an exacerbation of COPD may hinder the diagnosis of acute (coexisting) PE. The prevalence of PE among patients with a clinical diagnosis of acute exacerbation of COPD ranged from 3.3 to $29.1 \%$ in mostly small studies, ${ }^{5}$ and it is well-known that the specificity of D-dimers is too low to exclude PE in patients with concomitant chronic inflammatory diseases. Lastly, the bleeding risk of therapeutic anticoagulation will result in an additional burden of clinically significant bleeding events, which also detrimentally affect the overall prognosis.

\section{VTE, COPD, Physical Activity, and Quality of Life}

Whereas the impact on mortality is clear from several studies, coexisting COPD and VTE may also reinforce the detrimental impact on the health-related quality of life (HRQoL). Activityrelated dyspnea and exercise intolerance are key features of COPD and ultimately lead to reduced habitual physical activity, which significantly impair patients' quality of life. Psychological symptoms such as depression and anxiety are also associated with severe stages of COPD. ${ }^{6}$

Physical activity reduces the risk, delays the onset, or lessens the severity of many side effects of aging. ${ }^{7}$ Individual and regular physical education reduce mortality in both healthy adults and adults with chronic pulmonary and other diseases. ${ }^{8}$ As COPD is not reversible and lung function will not improve, HRQoL is a key, modifiable patient-centered factor for health outcomes. ${ }^{9}$

Also, for many VTE patients, impaired HRQoL is an important marker for clinically relevant outcomes such as overall mortality. Factors associated with below average HRQoL are unemployment, current depression and/or anxiety, anticoagulant-related bleeding, and difficulty getting VTE care. Potentially suitable interventions include early management of comorbid anxiety and depression, and making specific emotional support and care access resources available to VTE patients. ${ }^{10}$

The authors of the Tromsø study conclude that physical inactivity did not influence the mortality of COPD and VTE patients. Certain factors may have influenced the Tromsø study results. The symptom burden of the diseases, including respiratory symptoms (such as progressive dyspnea), fatigue, impaired physical performance, decreased physical activity, and reduced HRQoL are all consequences of the underlying conditions and depend on how the individual adapts to and manages their disease. The dose and frequency of specific pharmacological treatments, adherence to physical activity, breathing exercises, or complementary yoga, all play a role in reducing symptoms. ${ }^{11}$ Other adjunctive nonpharmacological treatments may also improve HRQoL in patients with VTE and COPD. ${ }^{12}$ The beneficial aspects of physical activity and other nonpharmacological treatments on quality of life could be investigated further in connection with VTE in combination with COPD. The aging population of patients with VTE and COPD increasingly has additional cardiovascular or

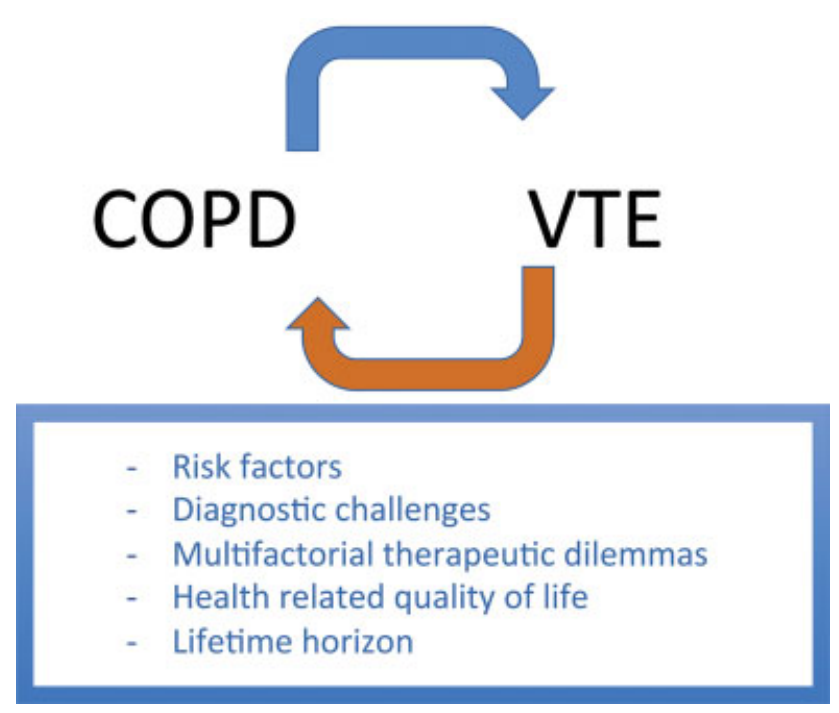

Fig. 1 Challenging intertwinements of chronic obstructive pulmonary disease (COPD) and venous thromboembolism (VTE).

malignant diseases. The beneficial effect of moderate physical activity and nonpharmacological interventions need to be objectively analyzed to determine the beneficial effect on quality of life in these patient groups (-Fig. 1).

\section{What the Future May Offer}

Even when the coexisting severe COPD drives the overall mortality in severe COPD, it is important to remind that VTE may be a tipping point tilting the prognosis of these severely ill patients. Recently, the importance of concomitant diseases for the prediction of VTE recurrence and mortality was highlighted. ${ }^{2}$ The dynamic assessment of risk factors over the lifetime predicted the risk for VTE more accurately than analysis at a fixed time point. ${ }^{13}$ The dangerous liaisons between VTE and COPD can be tackled by many interventions and the benefit over the lifetime horizon requires methodologically profound investigations.

Conflict of Interest

None declared.

\section{References}

1 Ageno W, Haas S, Weitz JI, et al; GARFIELD-VTE investigators. Characteristics and management of patients with venous thromboembolism: the GARFIELD-VTE Registry. Thromb Haemost 2019;119(02):319-327

2 Khorana AA. Modeling complexity: the case of cancer-related venous thromboembolism. Thromb Haemost 2019;119(11):1713-1715

3 Børvik T, Brækkan S, Evensen L, et al. Chronic obstructive pulmonary disease and risk of mortality in patients with venous thromboembolism - the Tromsø Study. Thromb Haemost 2020;120(03):477-483

4 Couturaud F, Bertoletti L, Sanchez O, et al. Frequency pulmonary embolism (PE) in patients with an acute exacerbation of chronic obstructive Pulmonary disease (COPD). PEP prospective trial. Eur Resp J 2019;54:PA3637

5 Pourmand A, Robinson H, Mazer-Amirshahi M, Pines JM. Pulmonary embolism among patients with acute exacerbation of 
chronic obstructive pulmonary disease: implications for emergency medicine. J Emerg Med 2018;55(03):339-346

6 Hanania NA, O'Donnell DE. Activity-related dyspnea in chronic obstructive pulmonary disease: physical and psychological consequences, unmet needs, and future directions. Int J Chron Obstruct Pulmon Dis 2019;14:1127-1138

7 Chodzko-Zajko WJ, Proctor DN, Fiatarone Singh MA, et al; American College of Sports Medicine. American College of Sports Medicine position stand. Exercise and physical activity for older adults. Med Sci Sports Exerc 2009;41(07):1510-1530

8 Geidl W, Schlesinger S, Mino E, et al. Dose-response relationship between physical activity and mortality in people with noncommunicable diseases: a study protocol for the systematic review and meta-analysis of cohort studies. BMJ Open 2019;9 (09):e028653

9 Long H, Howells K, Peters S, Blakemore A. Does health coaching improve health-related quality of life and reduce hospital admis- sions in people with chronic obstructive pulmonary disease? A systematic review and meta-analysis. Br J Health Psychol 2019;24 (03):515-546

10 Erickson RM, Feehan M, Munger MA, Tak C, Witt DM. Understanding factors associated with quality of life in patients with venous thromboembolism. Thromb Haemost 2019;119(11):1869-1876

11 Reychler G, Poncin W, Montigny S, Luts A, Caty G, Pieters T. Efficacy of yoga, tai chi and qi gong on the main symptoms of chronic obstructive pulmonary disease: a systematic review. Respir Med Res 2019;75:13-25

12 Hsieh PC, Yang MC, Wu YK, et al. Acupuncture therapy improves health-related quality of life in patients with chronic obstructive pulmonary disease: a systematic review and meta-analysis. Complement Ther Clin Pract 2019;35:208-218

13 Horvei LD, Brækkan SK, Hansen JB. Weight change and risk of venous thromboembolism: the Tromsø Study. PLoS One 2016;11 (12):e0168878 